\title{
Editorial
}

\section{Integration of Sensors in Control and Automation Systems}

\author{
Rafael Morales-Herrera, ${ }^{1}$ Antonio Fernández-Caballero, ${ }^{1}$ \\ José A. Somolinos, ${ }^{2}$ and Hebertt Sira-Ramírez ${ }^{3}$ \\ ${ }^{1}$ Escuela de Ingenieros Industriales de Albacete, Universidad de Castilla-La Mancha, 02071- Albacete, Spain \\ ${ }^{2}$ Escuela de Ingenieros Navales, Universidad Politécnica de Madrid, Madrid, Spain \\ ${ }^{3}$ Center for Research and Advanced Studies, National Polytechnic Institute, Mexico City, Mexico \\ Correspondence should be addressed to Rafael Morales-Herrera; rafael.morales@uclm.es
}

Received 9 January 2017; Accepted 9 January 2017; Published 12 March 2017

Copyright (C) 2017 Rafael Morales-Herrera et al. This is an open access article distributed under the Creative Commons Attribution License, which permits unrestricted use, distribution, and reproduction in any medium, provided the original work is properly cited.

Control theory is an interdisciplinary branch of engineering and mathematics dealing with the behavior of dynamic systems with inputs. The objective of control theory is to calculate solutions for the proper corrective action from the controller that results in system stability and improved performance. Automation and Industrial Control Systems (ICS) encompass many applications and uses of industrial and facility control and automation systems. ICS are defined by ISA-99/IEC 62443 as "a collection of personnel, hardware, and software that can affect or influence the safe, secure, and reliable operation of an industrial process."

Control systems are composed by five main elements: sensors, transducers, transmitters, controllers, and final control elements or actuators. This special issue focuses on sensors and, more concretely, on sensor integration in automation and control systems. Let us remind you that a sensor is defined as a device that converts a physical stimulus into a readable output. The role of a sensor in a control and automation system is to detect and measure some physical effect, providing this information to the control system.

The integration of sensors in control and automation systems has received a great deal of attention from a considerable number of researchers and from the industrial community in the last years. Emphasis is placed on the importance of creating improvements in control and automation systems in order to meet the challenges of developing and refining new applications. These systems have to integrate a variety of sensory information and human knowledge for the sake of efficiently carrying out tasks with or without human intervention.
In fact, the integration of sensors into intelligent devices and systems has increased the capacity to measure, analyze, and aggregate data at a localized level. Autonomous and connected sensors are able to selectively sample and measure many physical properties. Built on the increasing capabilities of fixed-access and wireless networks, smart sensor developments allow the collection of raw data, which is processed into information and conveyed via a network connection.

The concept of sensor integration is close to the sensor fusion term, which is defined as "the art of processing data from multiple sensors with an aim to replicate a physical environment or induce intelligence to control a phenomenon with increased precision and reliability." Sensor fusion or integration is evolving rapidly as the basis of robust control systems that can make sense of imperfect input despite the environment in which it operates. Data from multiple sensors are fused to increase response and accuracy, delivering control systems that until recently could only be theorized, drawing on such techniques as artificial intelligence, pattern recognition, digital signal processing, and statistical estimation. Moreover, recent advances in sensor technology and processing techniques, combined with improved hardware, make real-time fusion of data possible.

This special issue was aimed at exhibiting the latest research achievements, findings, and ideas in the integration of sensors in control and automation systems. The topics faced in this special issue were as follows: sensor systems for control and automation: sensors and sensor networks, intelligent sensors, sensor uncertainty for fault tolerant control, distributed and multimodality sensor network for control 
and automation, and so on; control: adaptive control, robust control, active disturbance rejection control, complex systems, identification and estimation, nonlinear systems, intelligent systems, sensor networks, delay systems, precision motion control, control applications, and so on; automation: man-machine interactions, process automation, networkbased systems, intelligent automation, planning, scheduling and coordination, and so on; robotics: modelling and identification, mobile robotics, mobile sensor networks, perception systems, visual servoing, robot sensing and data fusion, and so on; process based control: sensor development, system design, and control development; control and automation systems: fault detection and isolation, sensing and data fusion, flight control and surveillance systems, rescue and field robotics, guidance control systems, industry, military, space and underwater applications, linear and nonlinear control systems, signal and image processing, and so on; industrial informatics: embedded systems for monitoring and controlling.

\section{Acknowledgments}

We would like to thank all the authors for their excellent contributions and also the reviewers for their valuable help. We would also like to thank all members of the editorial board for approving this special issue.

Rafael Morales-Herrera Antonio Fernández-Caballero José A. Somolinos Hebertt Sira-Ramírez 


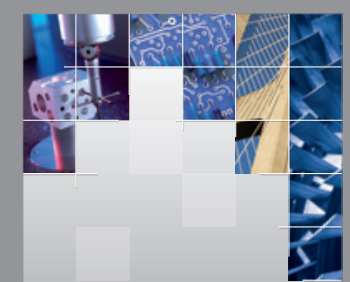

\section{Enfincering}
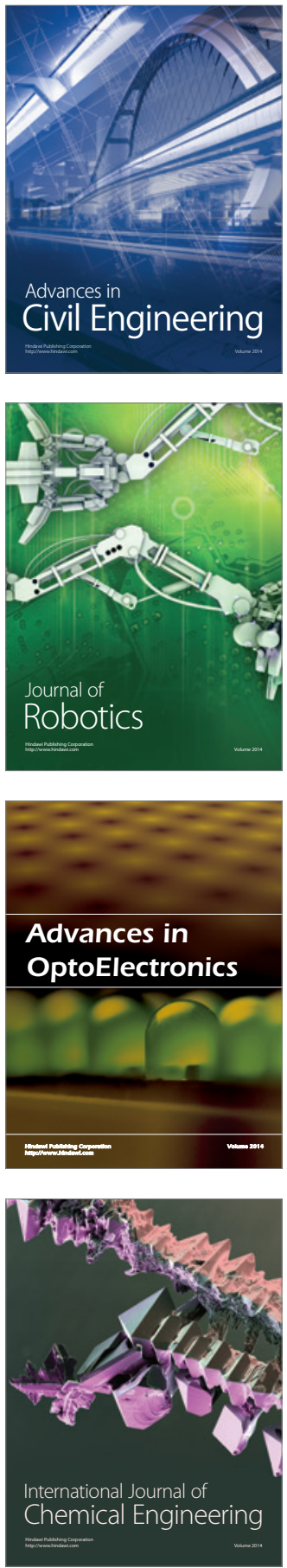

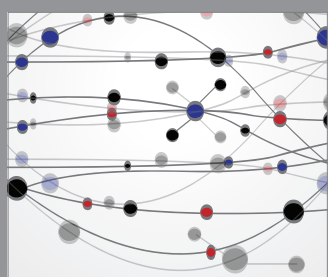

The Scientific World Journal

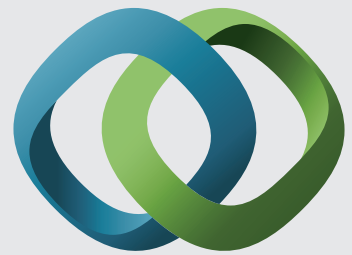

\section{Hindawi}

Submit your manuscripts at

https://www.hindawi.com
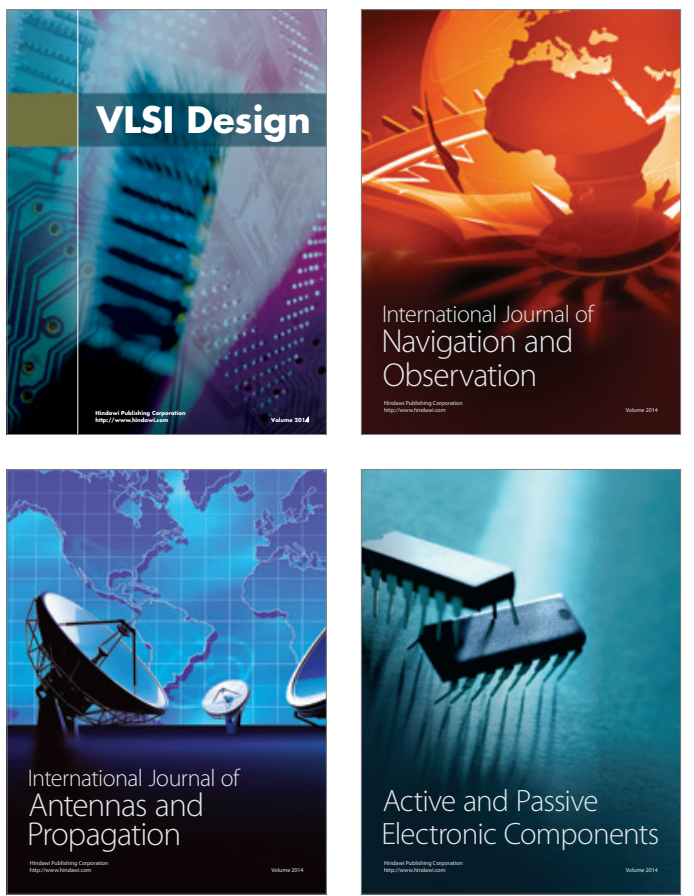
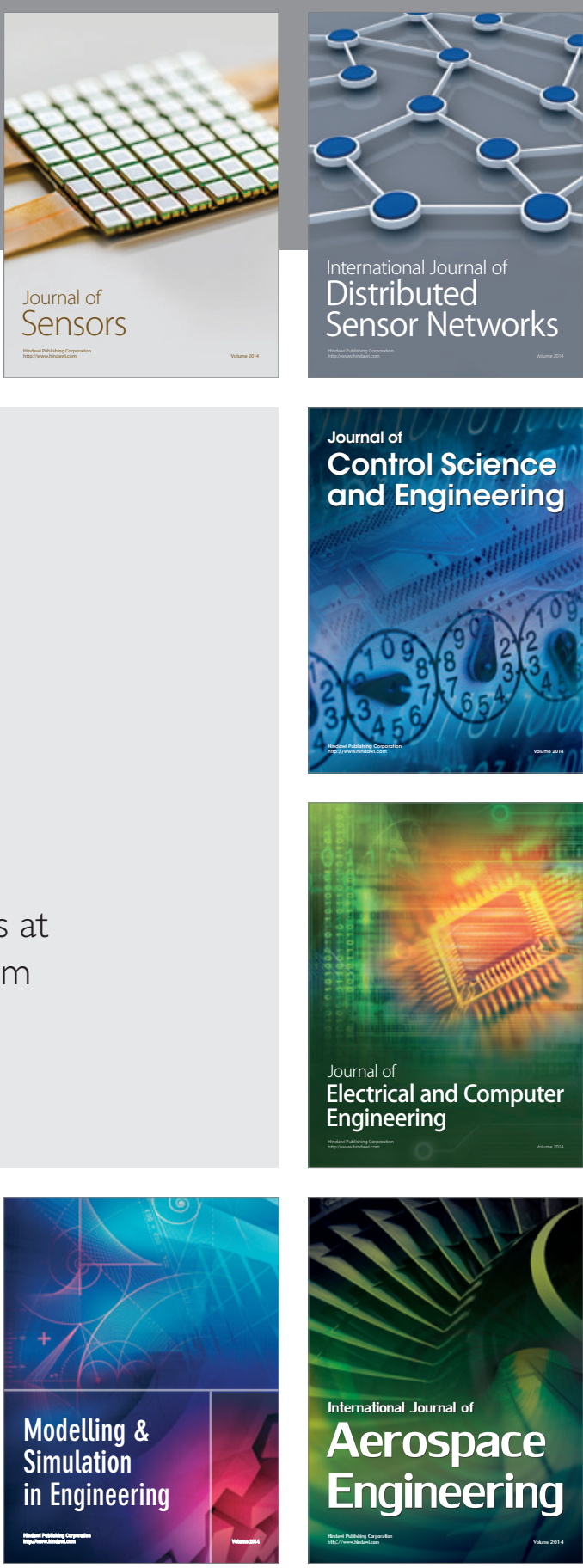

International Journal of

Distributed

Sensor Networks

$-$

Joumal of

Control Science

and Engineering
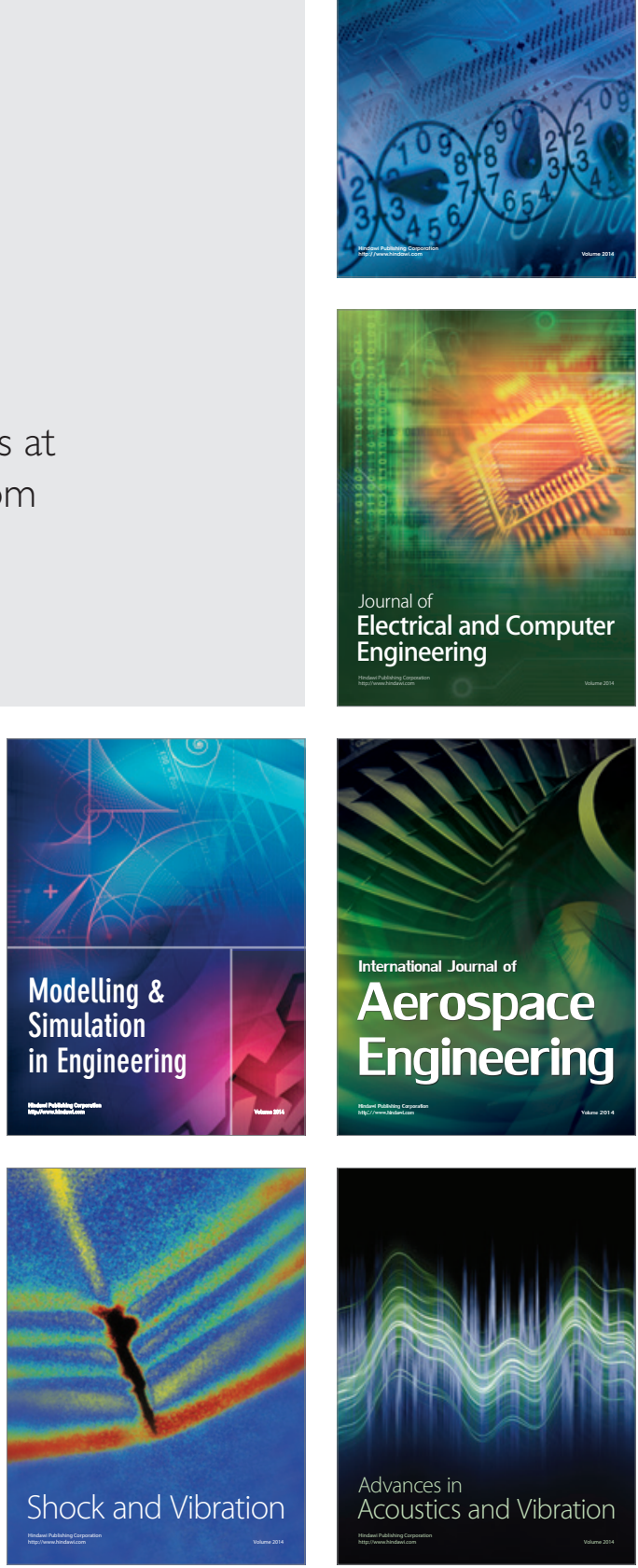